Психология. Журнал Высшей школы экономики,

2017. T. 14. № 3. C. 433-452. DOI: 10.17323/1813-8918-2017-3-433-452

\title{
СОСТОЯТЕЛЬНОСТЬ КАК ПСИХОЛОГИЧЕСКИЙ КОНСТРУКТ: ОПЫТ ПСИХОМЕТРИЧЕСКОЙ ВАЛИДИЗАЦИИ
}

\author{
Д.А. ЛЕОНТЬЕВ ${ }^{\mathrm{a}}$, Л.С. КИФАК ${ }^{\mathrm{b}}$, О.В. МИТИНА \\ В.А. ПЕТРОВСКИЙ ${ }^{\mathrm{a}}$ Ж.С. ТИХОБРАЗОВА
}

${ }^{2}$ Напиональный исследовательский университет «высиая икола экономики», 101000, Россия, Москва, ул. Мясницкая, д. 20

${ }^{b}$ МГУ имети М.В. Ломоносова, 119991, Россия, Москва, Летииские горы, д. 1

\begin{abstract}
Резюме
Цель данного исследования - психометрическая апробация показателей методики «Психологическая состоятельность личности» (ПСЛ) В.А. Петровского, построенной на основе авторской метаимпликативной модели экзистенциального выбора с использованием формул булевой алгебры (Петровский, 2013). Исследование было проведено на двух выборках: студентах 1-го курса факультета психологии $(\mathrm{N}=88)$ и девушках - профессиональных моделях $(\mathrm{N}=57)$, близких по социально-демографическим характеристикам, но радикально различающихся по образу жизни и вытекающцм из него требованиям к саморегуляции личности. Помимо методики «Психологическая состоятельность личности» использовались также традиционные психометрические методики личностного потенциала: Шкала удовлетворенности жизнью Э. Динера, Общий опросник самоэффективности Р. Шварцера и др. Обработка результатов включала в себя сравнение шести показателей методики для двух разных типов задач, задаваемых инструкцией, а также для двух выборок; дисперсионный анализ влияния этих двух переменных на показатели ПСЛ и корреляционный анализ связей показателей методики ПСЛ с показателями двух дополнительных методик. Установлено, что в выборке моделей ни один из показателей ПСЛ не различается значимо для двух типов задач, в то время как в выборке студенток значимые (в большинстве случаев высокозначимые) различия обнаруживаются для всех показателей, кроме доступности задачи. У студенток корреляции всех показателей ПСЛ с удовлетворенности жизнью значимо не различаются для двух типов задач. Интенция и волюнтаризм у них связаны с удовлетворенностью жизнью положительно, здравомыслие и самоконтроль отрицательно, а доступность и установка - незначимо. У моделей значимых корреляций существенно меньше; исключение - значимая положительная корреляция со здравомыслием и отрицательная с волюнтаризмом для «задачи по вкусу». Полученные данные позволяют говорить об эвристичности феноменологической модели состоятельности и о конструктной валидности методики ПСЛ.
\end{abstract}

Ключевые слова: саморегуляция, психологическая состоятельность, метаимпликативная модель, удовлетворенность жизнью, самоэффективность.

Работа выполнена при поддержке Российского научного фонда, проект № 16-18-10439 «Системно-динамический анализ регуляции деятельности». 
На данный момент понятия регуляции и саморегуляции все чаще обсуждаются в мировой психологической литературе. Можно говорить о ключевой роли этих процессов для всего психологического знания. Понятия регуляции и саморегуляции характеризуют динамику поведения сложных целенаправленных систем управления параметрами системы в соответствии с внутренними критериями и актуальным состоянием внешней среды. В основе этого управления лежит регулярно поступающая в реальном времени информация о текущих параметрах самой системы и об окружающей среде, с которой эта система взаимодействует. Понятие регуляции объединяет две тесно взаимосвязанные стороны одного процесса: это поддержание стабильности, самосохранение системы и ее адаптация к внешним условиям.

Общепринято выделение пяти основных элементов любого контура регуляции: сам процесс, критерии, в соответствии с которыми оценивается его ход, механизм обратной связи, который обеспечивает систему информацией об актуальном состоянии процесса, механизм сличения текущего состояния с критериями и управляющее воздействие на процесс с целью восстановить соответствие критерию (подробнее см.: Леонтьев, 2006, 2011). О саморегуляции мы говорим тогда, когда система регулирует сама себя, реагируя на отклонения процесса от заданных параметров. Если же источник управляющего воздействия находится вне самого процесса, речь идет о регуляции.

Обзор многочисленных исследований саморегуляции в современной психологии не входит в задачи данной работы (см., в частности: Carver, Sheier, 1998; Леонтьев, 2011; Моросанова, 2012; и др.). Данное исследование направлено на проверку следствий, вытекающих из одной конкретной модели, а именно из модели В.А. Петровского (2010, 2013).

\section{Феноменологическая модель саморегуляции В.А. Петровского и конструкт психологической состоятельности}

В рассматриваемой концепции состоятельность определяется как средоточие возможностей, побуждающих активность. Состоятельность объединяет два аспекта: достижения человека и стремление воспользоваться ими; с одной стороны результаты действия, с другой импульсы к его продолжению.

Автор модели предложил под этим углом зрения интерпретацию модели выбора риска Дж. Аткинсона, эффекта «настойчивого поведения» Н. Физера, гипотетических феноменов рефлексивной возгонки влечения и оптимума в соотношении доверия и контроля («доверяй, но проверяй»), взлетов и падений в переживаниях влюбленных и др. (Петровский, 2013). Анализируя известную модель выбора риска Дж. Аткинсона (Atkinson, 1957), В.А. Петровский показал, что предложенная модель совершенной саморегуляции дает более точный прогноз выборов уровня трудности задач и имеет более простое строение, чем модель Аткинсона.

На основе этой модели ее автором была разработана методика «Психо- 
логическая состоятельность личности».

Предполагается, что ощутить свою состоятельность человеку позволяет участие в решении задач различного рода. В настоящем исследовании использовались задачи двух типов: «Задача дня» и «Задача по вкусу». Задачи дня относятся к категории повседневных задач; говорят, что такие задачи ставит перед человеком «сама жизнь»; чтобы взяться за решение этих задач, от человека требуется согласие. Задачи по вкусу - ставит перед собой человек сам; они полностью отвечают его склонностям и интересам.

В методику включены два опросника, соответствующие двум названным типам задач. Содержание вопросов в «Задаче дня» и «Задаче по вкусу» идентично, различие заключается лишь в инструкции, предлагаемой человеку перед заполнением. Подчеркивается, что в одном случае речь идет о задаче, выбранной и поставленной самостоятельно (задача по вкусу), а в другом - об одной из задач, которые могут возникать перед человеком в силу внешних причин (задача дня) (см. Приложение).

В начале опросника поставлен общий вопрос: «Как часто при решении такой задачи вы...», а далее следует восемь утверждений, касающихся частоты переживаний и мыслей, сопутствующих решению такой задачи; ответ испытуемого - это метка на графической шкале «редко - часто». При обработке ответов метка, поставленная в начало шкалы (крайнюю левую точку), соответствует значению 0\%, а в конец шкалы (крайнюю правую точку) - значе- нию 100\% (промежуточные значения вычисляются в процентах как результаты деления длины отрезка от начала шкалы до метки на обшую длину шкалы).

Утверждения составлены таким образом, что в них зашифрованы 3 элемента состоятельности: «интенция» (х), «доступность задачи» (у) и «иррациональные ожидания» («вера в чудо») (s). Возможные проявления каждого из элементов - наличие или отсутствие. Каждый из элементов представлен позитивным (наличие) или негативным (отсутствие) полюсом; наличие или отсутствие маркируется соответственно через приписываемые значения 1 и 0.

O разных аспектах состоятельности дают представление восемь регуляторных состояний, отображенных в таблице 1 (названия регуляторных состояний несколько изменены по сравнению с более ранними: Петровский, 2013). В таблице для каждого регуляторного состояния указана выраженность каждого элемента состоятельности, которая задается дискретно, посредством бинарной переменной, принимающей значения $\{0,1\}$.

Предполагается, что уровень проявлений этих элементов в продвижении к успеху определяется частотой их присутствия в сознании. Иными словами, речь могла бы идти о выраженности присутствия в сознании склонности действовать, прогнозировать доступность задачи, надеяться на успех. Эмпирически этому соответствует то, насколько часто, в представлении субъекта, он действует, подчиняясь мотиву (х), ощущает доступность желаемого (у), верит за пределами рационального расчета (s). 
Таблица 1

Вопросы методики, соответствующие им регуляторные состояния и выраженность трех основных элементов состоятельности

\begin{tabular}{|c|c|c|c|c|c|}
\hline № & $\mathbf{x}$ & $\mathbf{y}$ & $\mathbf{s}$ & $\begin{array}{l}\text { Регуляторное } \\
\text { состояние }\end{array}$ & Формулировка вопроса методики \\
\hline $\mathrm{f} 1$ & 0 & 0 & 0 & «в тупике» & $\begin{array}{l}\text { 7. Вам не хочется ничего делать, нет никаких идей, } \\
\text { не испытываете ничего, кроме ощущения полной } \\
\text { бесперспективности? }\end{array}$ \\
\hline $\mathrm{f} 2$ & 0 & 0 & 1 & «авось» & $\begin{array}{l}\text { 6. Отказываетесь решать задачу по-старому, } \\
\text { «отстраиваетесь» от ситуации, внутренне } \\
\text { подготавливаетесь к новому решению } \\
\text { («переключаетесь»)? }\end{array}$ \\
\hline $\mathrm{f} 3$ & 0 & 1 & 0 & $\begin{array}{l}\text { «ответ в конце } \\
\text { задачника» }\end{array}$ & $\begin{array}{l}\text { 4. Думаете: вот бы пришел кто-нибудь и сказал, что } \\
\text { делать дальше (да только, увы, не будет этого)? }\end{array}$ \\
\hline $\mathrm{f} 4$ & 0 & 1 & 1 & «сама пойдет» & $\begin{array}{l}\text { 2. Считаете нужным дождаться «того самого» } \\
\text { счастливого случая, когда ситуация сама } \\
\text { подскажет, как быть и что делать (полагаетесь на } \\
\text { удачное стечение обстоятельств, как бы } \\
\text { наталкивающее на открытие)? }\end{array}$ \\
\hline $\mathrm{f} 5$ & 1 & 0 & 0 & «вопреки» & $\begin{array}{l}\text { 3. Исходите из необходимости «отмести» версии, } \\
\text { кажущиеся вам сомнительными? }\end{array}$ \\
\hline f6 & 1 & 0 & 1 & $\begin{array}{l}\text { «ищите и } \\
\text { обрящете» }\end{array}$ & $\begin{array}{l}\text { 1. Перепроверяете себя, пытаетесь удостовериться } \\
\text { в правильности своих догадок и предположений } \\
\text { (как бы «испытывая их на прочность»)? }\end{array}$ \\
\hline $\mathrm{f} 7$ & 1 & 1 & 0 & $\begin{array}{c}\text { «забудь } \\
\text { сомнения» }\end{array}$ & $\begin{array}{l}\text { 8. Думаете: «Надо попробовать!», хотя и } \\
\text { чувствуете, что это совершенно напрасно? }\end{array}$ \\
\hline 18 & 1 & 1 & 1 & $\begin{array}{l}\text { «вперед к } \\
\text { победе» }\end{array}$ & $\begin{array}{l}\text { 5. Верите в то, что не совершите ошибки, действуя } \\
\text { так, а не как-то иначе? }\end{array}$ \\
\hline
\end{tabular}

Примечание. № - № регуляторного состояния, $\mathrm{x}-$ «интенция» («буду»), у - «доступность» («посильно»), s - «иррациональные ожидания» («верю»).

$B$ результате «интенция» $(x)$ определяется тем, насколько часто испытуемый, по его мнению, склонен предпринимать активные действия, в которых присутствует элемент х (см. таблицу 1), ведущие к решению, чему соответствуют частота состояний «вопреки», «ищите и обрящете», «забудь сомнения», «вперед к победе». При подсчете суммируются субъективные частоты регуляторных состояний, в которых $\mathrm{x}=1: \mathrm{f}_{5}(1,0,0)$, $f_{6}(1,0,1), \quad f_{7}(1,1,0), \quad f_{8}(1,1,1)$. Эти состояния соответствуют вопросам 1, 3, 5, 8 методики. Далее определяется доля субъективной встречаемости позитивного полюса мотива действия из расчета: $\mathrm{x}=\left(\mathrm{f}_{5}+\mathrm{f}_{6}+\mathrm{f}_{7}+\mathrm{f}_{8}\right) / \sum_{1}^{8} \mathrm{f}_{\mathrm{i}}$, где $\sum_{1}^{8} \mathrm{f}_{\mathrm{i}}-$ сумма частот проявления всех состояний вообще.

$\mathrm{O} \ll$ «оступности задачи» $(y)$ мы судим по тому, насколько часто у 
испытуемых возникает ощущение, мысль, что «решение идет в руки» «ответ в конце задачника», «сама пойдет», «забудь сомнения», «вперед к победе» (чем чаще такая мысль возникает, тем субъективно доступнее решение, податливее задача), Так же, как и в случае оценки х, подсчитывается средняя доля субъективной встречаемости переживания доступности задачи: $\mathrm{y}=\left(\mathrm{f}_{3}+\mathrm{f}_{4}+\mathrm{f}_{7}+\mathrm{f}_{8}\right) / \sum_{1}^{8} \mathrm{f}_{\mathrm{i}}$. Эти состояния соответствуют вопросам 2 , 4, 5, 8 методики.

«Иррачиональнье ожидания» (s) (ничем не подтвержденная вера в то, что удастся найти решение) характеризуются частотой переживания таких состояний, как «авось», «сама пойдет», «ищите и обрящете», «вперед, к победе». Соответственно $\mathrm{s}=\left(\mathrm{f}_{6}+\right.$ $\left.+\mathrm{f}_{4}+\mathrm{f}_{8}+\mathrm{f}_{2}\right) / \sum_{1}^{8} \mathrm{f}_{\mathrm{i}}$. ЭТи состояния соответствуют вопросам 1, 2, 5, 6 методики.

Таким образом, три основных показателя определяются феноменологическим «весом» соответствующих переживаний по отношению ко всем актуальным переживаниям. Все они лежат в диапазоне от 0 до 1 (включая 0 - полное отсутствие выраженности данного элемента, 1 полное присутствие выраженности данного элемента).

На основе этих трех основных показателей вычисляются производ- ные показатели. Для вычислений используются формулы булевой алгебры. Так, например, на основании показателей а и b вычисляется формула a $\supset$ b. В булевой алгебре, где все переменные могут принимать значения 0 и 1 , эта операция называется материальной импликацией, и определяется она так, как представлено в таблице 2.

Таким образом, значение формулы на бинарных переменных будет равно нулю тогда и только тогда, когда интенция высокая (равна 1), а податливость низкая (равна 0). Это соответствует ситуации «хочу, но не могу». Во всех остальных случаях значение функции равно 1.

Мы предполагаем, что эта связка, выраженная в данном случае переменной F, обозначает установку как проявление «полевого» (К. Левин) управления действием без прямого участия сознания (Д.Н. Узнадзе), что выражается переживаниями «надо», «необходимо», «тянет». Человек при этом действует как бы на автопилоте.

Поскольку в булевой алгебре верно следующее равенство:

$$
F=x \supset y=1-x+x y
$$

(читатель может проверить это самостоятельно), то мы можем расширить

Таблица 2

Значение операции импликации для дихотомических переменных

\begin{tabular}{|c|c|c|}
\hline $\mathbf{a}$ & $\mathbf{b}$ & $\mathbf{a} \supset \mathbf{b}$ \\
\hline 1 & 1 & 1 \\
\hline 1 & 0 & 0 \\
\hline 0 & 1 & 1 \\
\hline 0 & 0 & 1 \\
\hline
\end{tabular}


эту формулу на область значений $\{\mathrm{x}, \mathrm{y}\} \in[0,1]$. Из этого следует, что с повышением доступности задачи усиливается установочная регуляция деятельности; при низкой интенции деятельность протекает без осознанной цели независимо от доступности задачи.

Если переписать формулу (1) в виде:

$$
F=1-x(1-y),
$$

то видим, что при высоком значении $\mathrm{x}$ (осознанное «хочу») снижается значение F (ощущение «надо»). При этом чем доступнее задача у, тем дольше сохраняет свои высокие значения $\mathrm{F}$.

Для элемента s - иррационального ожидания введем антонимичный ему элемент $\mathrm{z}=1-\mathrm{s}$.

Подобно остальным элементам, z лежит в диапазоне [0, 1]. Чем больше s - иррациональных ожиданий, тем меньше z. Вполне логично это переживание назвать здравомыслием.

Свяжем теперь импликацией установку и здравомыслие, введя показатель внутренней цензуры (самоконтроля) U:

$$
U=F \supset z
$$

Исходя из правил, представленных в таблице 2, установка, полностью основанная на иррациональных ожиданиях (в отсутствие здравомыслия), предполагает полное отсутствие самоконтроля ( $\mathrm{U}=0)$, а во всех остальных случаях U равна 1, т.е. высокий показатель здравомыслия в любом случае обеспечивает высокое значение $\mathrm{U}$, а при низком $\mathrm{F}$ самоконтроль будет высоким ( $\mathrm{U}=1)$ даже при низком здравомыслии.

Если теперь подставить в формулу (3) значения для $\mathrm{F}$ и преобразовать ее аналогично формуле (1), то получим:

$$
U=(x \supset y) \supset z=z+(1-z)(1-y) x^{1}
$$

C ростом интенции растет U, т.е. самоконтроль, задачей которого является сдерживание своих желаний. При этом скорость роста этого контроля уменьшается по мере увеличения доступности, с одной стороны, и здравомыслия - с другой. Эти два компонента вполне могут «компенсировать» необходимость самоконтроля.

Перепишем теперь формулу (4) следующим образом:

$$
U=z+x-z x-y x(1-z)
$$

Ее можно интерпретировать так: с ростом доступности необходимость во внутренней цензуре уменьшается, при этом чем больше интенция и меньше здравомыслие, тем быстрее это уменьшение. Чем больше здравомыслия, тем больше самоцензуры.

Еше один компонент переживания $\triangle=\mathrm{x}-\mathrm{U}$.

\footnotetext{
${ }^{1}$ Читателям, знакомым с рефлексивной теорией В.А. Лефевра (Лефевр, 1996, 2004), очевидна связь этого выражения с формулой «готовности субъекта к биполярному выбору»; преемственность и своеобразие модели состоятельности анализируется в книге В.А. Петровского «"Я” в персонологической перспективе» (Петровский, 2013).
} 
Формула отражает меру «подавления» желаний внутренним цензором. Чем меньше выраженность этого цензора (самоконтроля), тем больше значение $\triangle$. Логично назвать этот показатель волюнтаризмом. Он лежит в диапазоне $[-1,1]$. Если х $<\mathrm{U}$, то $\Delta<0$ - т.е. самоконтроль, подавляя желания, способствует снижению волюнтаризма. Минимальное значение $\Delta=-1$ реализуется при $\mathrm{x}=0$ и $\mathrm{U}=1$ (что возможно только при $\mathrm{z}=1$ : желаний нет, одно здравомыслие).

Феноменологическая модель предполагает, что все указанные показатели методики (три основных и три производных) действительно отражают определенные эффекты саморегуляции деятельности. Как следует из изложенного выше, логические следствия из исходных положений и вводных условий модели хорошо согласуются как со здравым смыслом, так и с психологическими знаниями. Однако этого недостаточно для верификации модели, поэтому с целью валидизации модели психологической состоятельности личности и феноменологической методики ее диагностики нами было проведено описываемое далее эмпирическое исследование.

Поскольку сама модель основана на чисто теоретических соображениях, мы поставили в настоящем исследовании задачу ее конструктной валидизации на основе сопоставления переживаний, возникающих в двух видах задач и у двух категорий респондентов. «Задача по вкусу» это задача, выбираемая самим респондентом в соответствии с его интенцией, индивидуальными особенностями и личными предпочтениями; «Задача дня» - это задача, которую социально-нормативный образ жизни (А.Г. Асмолов) вынуждает респондента постоянно выполнять вне зависимости от его интенции, индивидуальных особенностей и личных предпочтений. Две выборки участниц исследования составили студентки 1-го курса МГУ, образ жизни которых не предполагает особенного давления обстоятельств, напряженной целенаправленности и конкурентности, и близких к ним по возрасту и образовательному уровню девушек - профессиональных моделей, образ жизни которых предполагает повышенные требования к саморегуляции, высокую конкурентность и сильную зависимость от внешних постоянно меняющихся условий.

В частности, в числе следствий из модели, которые выступают эмпирически проверяемыми в нашем исследовании гипотезами, выступают следующие.

1. В выборке студенток показатели состоятельности для двух типов задач будут больше различаться, чем в выборке моделей, поскольку для них более ощутим разрыв между выбираемыми и навязываемыми занятиями.

2. У студенток при решении задачи по вкусу показатели интенции и волюнтаризма должны быть выше, а показатели здравомыслия и самоконтроля ниже, чем у девушек-моделей.

3. Показатели состоятельности, за исключением доступности, будут связаны с удовлетворенностью жизнью и с общей самоэффективностью. Эти связи будут более выражены у студенток по сравнению с моделями и в «задаче по вкусу» по сравнению с «задачей дня». 


\section{Методики и организация исследования}

Уиастниками исследования выступили 88 студенток 1-го курса факультета психологии МГУ имени М.В. Ломоносова и 57 профессионально работающих девушек-моделей со стажем профессиональной деятельности не менее двух лет, всего 145 человек, все женского пола. Возраст участников - в диапазоне от 17 до 35 лет. Средний возраст студенток составил 18 лет (стандартное отклонение - 0.6 года), средний возраст моделей 22 (стандартное отклонение - 3.6 года). Мы ставили задачу сравнить две контрастные группы - студенток, которых пока не слишком напрягают вопросы карьерных достижений и самоутверждения, и девушек, работаюших в напряженной высококонкурентной атмосфере с высокими требованиями к саморегуляции. Подразумевалась, что студентки обладают бо́льшим простором выбора решаемых задач, среди которых встречаются «задачи по вкусу», чем модели, чьи «вкусы» (предпочитаемые задачи) в значительной мере предопределяются необходимостью придерживаться строгого режима самоограничения, продиктованного «задачами дня».

Проверялись гипотезы о связях между параметрами, определяемыми методикой психологической состоятельности личности, с одной стороны, и показателями удовлетворенности жизнью и самоэффективности, с другой стороны.

Прочедура исследования. Участникам предъявлялся для заполнения набор методик, позволяющий оценить состоятельность личности, удовлетворенность жизнью и самоэффективность личности. Бланки каждый респондент заполнял индивидуально.

В исследовании были использованы:

1. Методика «Психологическая состоятельность личности» (ПСЛ) (В.А. Петровский). Подробное описание методики приведено выше.

2. «Шкала общей самоэффективности» (ШІОС) - опубликованная русскоязычная адаптация (Шварцер и др., 1996) шкалы общей самоэффективности Р. Шварцера (Schwartzer, 1993), измеряющей самоэффективность как генерализованное убеждение индивида в его способности достигать целей в различных ситуациях. Опросник содержит 10 утверждений, которые оцениваются по 4балльной шкале.

3. Методика «Шкала удовлетворенности жизнью» (ШУДЖ), адаптация (Д.А. Леонтьев, Е.Н. Осин) методики SWLS (Satisfaction With Life Scale) Э. Динера и др. (Diener et al., 1985). Опубликована частично (Леонтьев, 2004; Осин, Леонтьев, 2008). Содержит пять пунктов, оцениваемых по 7-балльной шкале. Единственный показатель оценивает степень интегральной оценки удовлетворенности жизнью в целом.

Обработка данных проводилась с помощью статистического пакета SPSS 20.

\section{Результаты и обсуждение}

Начнем с анализа данных методики ПСЛ. На основании выполнения методики мы имеем шесть измеряемых показателей (интенция, доступность, здравомыслие, установка, 
самоконтроль, волюнтаризм), определенных в подвыборках по двум независимым переменным. Первая это тип задачи («задача по вкусу» и «задача дня») с повторными измерениями (каждый респондент отвечал на вопросы, касающиеся обоих типов задач). Вторая переменная отражает характер деятельности и образ жизни (студентки и работающие девушки-модели), она разбивает данные на две подвыборки, каждая с двумя типами задач и шестью индикаторами. Все шесть показателей являются нормально распределенными в четырех подвыборках. В таблице 3 приведены их описательные статистики.

В последнем столбце представлены $p$-значения результатов сравне- ния двух разных типов задач и в той, и в другой выборке (значение по парному $t$-критерию Стьюдента для связанных выборок). В столбцах, содержащих средние, в ряде случаев в скобках стоят указания $p$-значений, это означает, что различия по выраженности данного показателя в подвыборках моделей и студенток статистически значимы.

Отметим, что в студенческой выборке значения всех показателей, за исключением показателя доступности, значимо различаются для разных типов задач, иными словами, заданное инструкцией условие выбора одного из двух типов задач оказало большое влияние на результаты. Напротив, в выборке моделей значения всех шести показателей для двух

Таблица 3

Средние и стандартные отклонения по шести показателям в ситуации «задачи дня» и «задачи по вкусу» в выборках студенток и моделей

\begin{tabular}{|c|c|c|c|c|c|}
\hline & \multicolumn{2}{|c|}{ «Задача дня» } & \multicolumn{2}{|c|}{ «Задача по вкусу» } & \multirow{2}{*}{$\begin{array}{c}p \text { для двух } \\
\text { типов задач }\end{array}$} \\
\hline & $\mathrm{M}$ & SD & $\mathrm{M}$ & SD & \\
\hline \multicolumn{6}{|l|}{ Студентки } \\
\hline Интенция & 0.576 & 0.100 & $0.664(p=0.001)$ & 0.097 & 0.000 \\
\hline Доступность & 0.481 & 0.092 & 0.478 & 0.115 & 0.727 \\
\hline Здравомыслие & 0.471 & 0.088 & $0.395(p=0.036)$ & 0.110 & 0.000 \\
\hline Установка & 0.702 & 0.074 & $0.653(p=0.007)$ & 0.095 & 0.000 \\
\hline Самоконтроль & 0.630 & 0.068 & 0.607 & 0.084 & 0.036 \\
\hline Волюнтаризм & -0.054 & 0.112 & $0.056(p=0.002)$ & 0.121 & 0.000 \\
\hline \multicolumn{6}{|l|}{ Модели } \\
\hline Интенция & 0.593 & 0.103 & 0.601 & 0.121 & 0.607 \\
\hline Доступность & 0.480 & 0.098 & 0.504 & 0.106 & 0.067 \\
\hline Здравомыслие & 0.453 & 0.088 & 0.433 & 0.098 & 0.114 \\
\hline Установка & 0.688 & 0.088 & 0.698 & 0.098 & 0.448 \\
\hline Самоконтроль & 0.626 & 0.067 & 0.607 & 0.079 & 0.066 \\
\hline Волюнтаризм & -0.033 & 0.104 & -0.005 & 0.108 & 0.062 \\
\hline
\end{tabular}


типов задач значимо не различаются. Это подтверждает высказанную выше гипотезу 1.

При сравнении двух выборок мы получили результаты, показывающие, что средние при решении «задачи дня» в двух выборках не отличаются, а у студенток различия проявились по четырем показателям из шести. При решении «задачи по вкусу» у них значимо выше интенция и волюнтаризм и ниже значения здравомыслия и установки. Таким образом, эти данные подтверждают гипотезу 2 лишь отчасти (по отношению к показателю интенции).

Также был проведен двухфакторный дисперсионный анализ, в котором в роли факторов выступали и принадлежность к одной из двух групп, и тип задачи, а зависимыми переменными служили показатели методики ПСЛ. Данный вид анализа позволяет учесть оба фактора в комплексе и оценить межфакторное взаимодействие, т.е. выявить структурно-динамические особенности соотношения между ресурсами участников исследования и особенностями задач разного типа.

По наличию взаимодействия двух факторов показатели разделились на две группы. Первую группу образовали показатели интенции, здравомыслия, установки, волюнтаризма, в которых взаимодействие факторов было явным и высокозначимым $(p<0.01$ во всех четырех случаях). Как видно, в частности, на рисунке 1a, в, г, е, у девушек-моделей оба показателя слабо различаются для двух типов задач, в то время как у студенток они значимо меняются для «задачи дня» по сравнению с «задачей по вкусу».
Во вторую группу вошли показатели «доступность задачи» и «самоконтроль», для которых взаимодействие факторов было незначимым. Показатель «самоконтроль» (рисунок 1д) в обеих выборках снижается при переходе от «задачи дня» К «задаче по вкусу». Для студенток это снижение значимо, для моделей значимость снижения ( $p=0.066$ ) несколько превышает приемлемый уровень. Показатель «доступность задачи» (рисунок 1б) у моделей для «задачи по вкусу» растет по сравнению с результатами студенток, однако эти различия в нашем исследовании выражены в лучшем случае на уровне тенденции.

Теперь перейдем к анализу соотношения между показателями методики ПСЛ, вычисленными в соответствии с алгоритмами исходной теоретической модели, и показателями других методик, сделанных по традиционной психометрической методологии.

При вычислении итоговых показателей по шкалам Удовлетворенности жизнью и Самоэффективности была проверена согласованность пунктов с помощью показателя альфа Кронбаха. В первом случае согласованность равна 0.820 , во втором 0.851, что служит косвенным подтверждением репрезентативности данной выборки. У моделей удовлетворенность жизнью была выше, чем у студенток (по критерию Стьюдента $p=0.02)$, различий в самоэффективности обнаружено не было.

Для проверки гипотез 1 и 2 был проведен корреляционный анализ показателей методики психологической состоятельности личности для 
Рисунок 1

Графики выраженности показателей ПСЛ в зависимости от типа задачи в подвыборках моделей и студенток
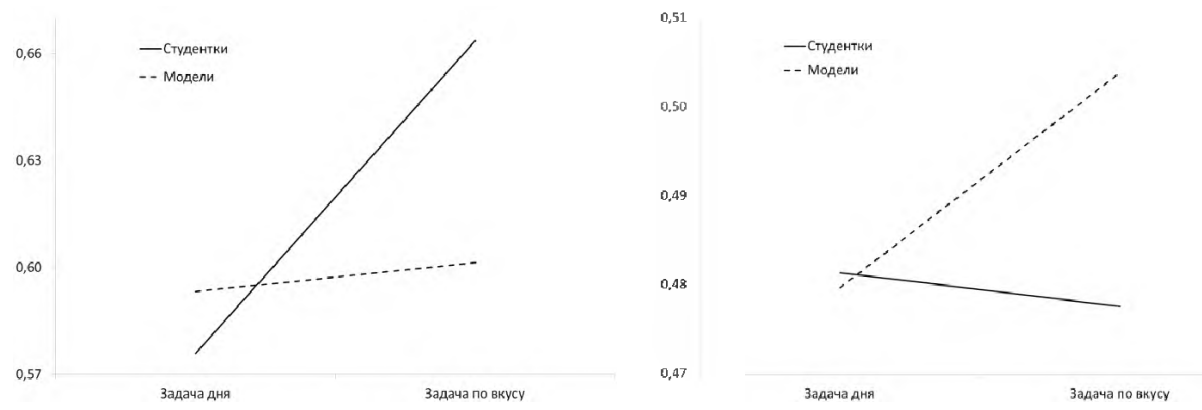

а) интенция, $p$-значение межфакторного взаимодействия $<0.001$

б) доступность, $p$-значение межфакторного взаимодействия $=0.103$
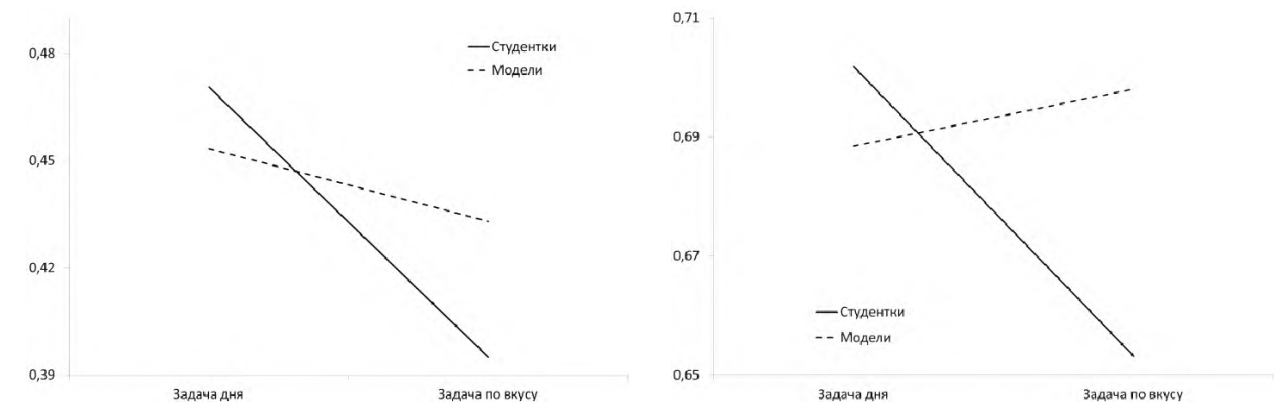

в) здравомыслие, $p$-значение межфакторного взаимодействия $=0.003$

г) установка, $p$-значение межфакторного взаимодействия $=0.001$
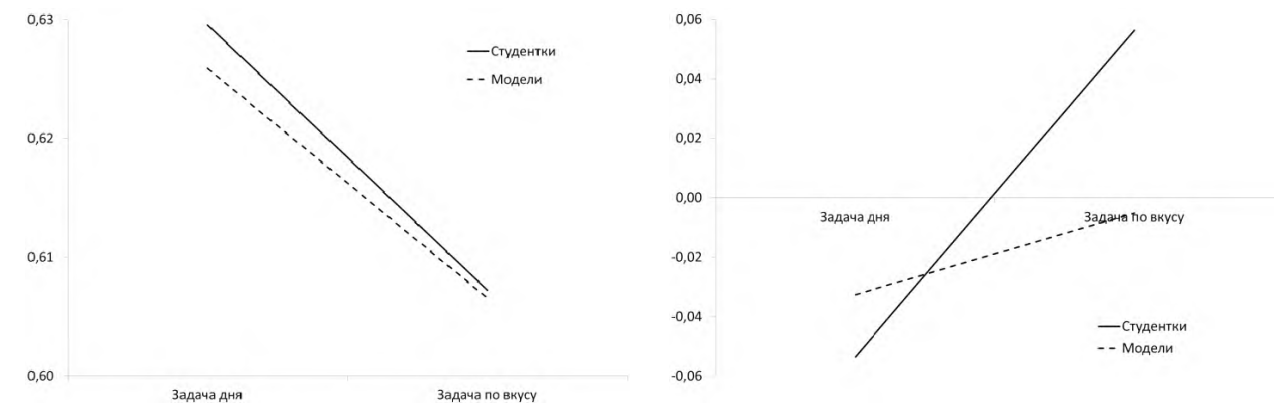

д) самоконтроль, $p$-значение межфакторного взаимодействия $=0.847$

е) волюнтаризм, $p$-значение межфакторного взаимодействия $<0.001$ 
двух типов задач и дополнительных методик, выявляющих удовлетворенность жизнью и самоэффективность. Использовалась традиционная линейная корреляция Пирсона.

В таблицах 4 и 5 приведены значения корреляций показателй ПСЛ с удовлетворенностью жизнью и самоэффективностью для двух задач и для двух выборок, а также значимость различий между значениями корреляций для двух типов задач (в последнем столбце $p$ ) и различий в коэффициентах корреляции в разных выборках (в верхних частях таблиц).

Как видно из таблицы 4, у студенток корреляции всех показателей ПСЛ с удовлетворенности жизнью значимо не различаются по двум типам задач. Интенция и волюнтаризм у них связаны с удовлетворенностью жизнью положительно, здравомыслие и самоконтроль - отрицательно, а доступность и установка незначимо.

У моделей значимых корреляций существенно меньше. Корреляции всех показателей ПСЛ по «задаче дня» с удовлетворенностью жизнью незначимы, а по «задаче по вкусу» обнаруживаются значимая положительная корреляция со здравомыслием и отрицательная с волюнтаризмом, что противоположно по знаку соответствующим корреляциям у студенток. У моделей также большинство значений корреляций не различается для двух типов задач.

Коэффициенты корреляции удовлетворенности жизнью с показателями ПСЛ

Таблица 4

\begin{tabular}{|c|c|c|c|c|c|c|c|}
\hline & \multicolumn{3}{|c|}{ «Задача дня2 } & \multicolumn{3}{|c|}{ «Задача по вкусу» } & \multirow{2}{*}{$\begin{array}{c}p \text { между } \\
\text { задачами }\end{array}$} \\
\hline & $r$ & $\begin{array}{c}p \text { между } \\
\text { группами }\end{array}$ & $p$ & $r$ & $\begin{array}{c}p \text { между } \\
\text { группами }\end{array}$ & $p$ & \\
\hline \multicolumn{8}{|l|}{ Студентки } \\
\hline Интенция & 0.258 & & 0.015 & 0.234 & 0.062 & 0.028 & 0.870 \\
\hline Доступность & 0.169 & & 0.115 & -0.026 & & 0.813 & 0.201 \\
\hline Здравомыслие & -0.377 & 0.026 & 0.000 & -0.355 & 0.000 & 0.001 & 0.868 \\
\hline Установка & -0.049 & & 0.652 & -0.128 & & 0.236 & 0.604 \\
\hline Самоконтроль & -0.312 & 0.035 & 0.003 & -0.209 & 0.014 & 0.051 & 0.471 \\
\hline Волюнтаризм & 0.418 & 0.064 & 0.000 & 0.332 & 0.001 & 0.002 & 0.513 \\
\hline \multicolumn{8}{|l|}{ Модели } \\
\hline Интенция & 0.152 & & 0.258 & -0.086 & & 0.523 & 0.212 \\
\hline Доступность & -0.004 & & 0.979 & -0.042 & & 0.755 & 0.841 \\
\hline Здравомыслие & -0.008 & & 0.950 & 0.295 & & 0.026 & 0.105 \\
\hline Установка & -0.081 & & 0.548 & 0.069 & & 0.611 & 0.435 \\
\hline Самоконтроль & 0.045 & & 0.740 & 0.214 & & 0.110 & 0.370 \\
\hline Волюнтаризм & 0.122 & & 0.364 & -0.255 & & 0.056 & 0.046 \\
\hline
\end{tabular}


Единственное исключение - волюнтаризм, который при выполнении «задачи по вкусу» отрицательно связан с удовлетворенностью жизнью на грани приемлемого уровня значимости, а при выполнении «задачи дня» положительно, но незначимо

Если значимые корреляции с удовлетворенностью жизнью (таблица 4) в большей степени определялись спецификой выборки, то ситуация с самоэффективностью иная. Различия в корреляциях у студенток чаще определяются типом задачи, у моделей такое влияние меньше (хотя тоже есть). Мы видим в таблице 5, что у студенток интенция и волюнтаризм положительно коррелируют с самоэффективностью для «задачи дня», здравомыслие, самоконтроль, установка - отрицательно, а для «задачи по вкусу» значимые связи обнаруживают только интенция (положительная) и установка (отрицательная). $\mathrm{y}$ моделей значимые различия для двух типов задач обнаруживает только показатель волюнтаризма: хотя его противоположные по знаку корреляции с самоэффективностью незначимы, они значимо различаются для двух типов задач. Значимых различий между выборками всего два: у студенток в «задаче по вкусу» интенция и волюнтаризм сильнее по сравнению с моделями связаны с самоэффективностью.

Указанные значимые корреляции и различия в корреляциях в зависи-

Таблица 5

Коэффициенты корреляции самоэффективности с показателями ПСЛ

\begin{tabular}{|c|c|c|c|c|c|c|c|}
\hline & \multicolumn{3}{|c|}{ «Задача дня» } & \multicolumn{3}{|c|}{ «Задача по вкусу» } & \multirow{2}{*}{$\begin{array}{l}p \text { между } \\
\text { задачами }\end{array}$} \\
\hline & $r$ & $\begin{array}{c}p \text { между } \\
\text { группами }\end{array}$ & $p$ & $r$ & $\begin{array}{c}p \text { между } \\
\text { группами }\end{array}$ & $p$ & \\
\hline \multicolumn{8}{|l|}{ Студентки } \\
\hline Интенция & 0.350 & & 0.001 & 0.245 & 0.096 & 0.021 & 0.452 \\
\hline Доступность & 0.085 & & 0.430 & -0.122 & & 0.259 & 0.176 \\
\hline Здравомыслие & -0.346 & & 0.001 & -0.092 & & 0.392 & 0.080 \\
\hline Установка & -0.182 & & 0.090 & -0.213 & & 0.046 & 0.832 \\
\hline Самоконтроль & -0.216 & & 0.043 & 0.070 & & 0.517 & 0.059 \\
\hline Волюнтаризм & 0.442 & & 0.000 & 0.147 & 0.048 & 0.173 & 0.033 \\
\hline \multicolumn{8}{|l|}{ Модели } \\
\hline Интенция & 0.269 & & 0.045 & -0.042 & & 0.761 & 0.102 \\
\hline Доступность & -0.153 & & 0.261 & -0.253 & & 0.060 & 0.588 \\
\hline Здравомыслие & -0.109 & & 0.424 & 0.131 & & 0.334 & 0.214 \\
\hline Установка & -0.230 & & 0.088 & -0.105 & & 0.440 & 0.508 \\
\hline Самоконтроль & 0.066 & & 0.629 & 0.202 & & 0.136 & 0.476 \\
\hline Волюнтаризм & 0.223 & & 0.098 & -0.196 & & 0.148 & 0.028 \\
\hline
\end{tabular}


мости от типа задачи выборки хорошо согласуются с другими полученными данными.

\section{Заключение и выводы}

Основной задачей данного исследования была психометрическая апробация методики исследования психологической состоятельности личности и валидизация традиционными методами ее показателей, выведенных нетрадиционным путем логических импликаций с использованием элементов булевой алгебры. Сформулированные гипотезы были направлены на конструктную валидизацию методики путем сравнения ее показателей по двум типам задач в двух выборках с разным образом жизни и профессиональными требованиями.

Таблица 3 содержит данные, полностью подтверждающие гипотезу 1. В выборке моделей ни один из показателей значимо не различается по двум типам задач, в то время как в выборке студенток значимые (в большинстве случаев высокозначимые) различия обнаруживаются для всех показателей, кроме доступности задачи.

Вторая гипотеза также подтвердилась. Различия между двумя выборками для «задачи по вкусу» (но не для «задачи дня») были значимыми для четырех из шести показателей состоятельности, причем показатели интенции и волюнтаризма были выше у студенток, а показатели установки и здравомыслия у моделей (таблица 3).

Третья гипотеза подтвердилась отчасти (см. таблицы 4 и 5 ). Доступность задачи, как и предпола- галось, не связана с удовлетворенностью жизни и самоэффективностью независимо от выборки и типа задачи. Значимые корреляции с самоэффективностью обнаруживаются только у студенток и преимущественно по «задаче дня», где их обнаруживают почти все показатели, а по «задаче по вкусу» только показатели интенции и установки, однако различия между выборками практически везде незначимы. Связи с удовлетворенностью жизнью более многообразны, для «задачи по вкусу» они значимо различаются в двух выборках по четырем из шести показателей, причем во всех четырех случаях знак корреляции различается. Показатели состоятельности для «задачи дня» у моделей все незначимы, а у студенток они приобретают значимую выраженность в четырех случаях из шести. Таким образом, результаты подтвердили третью гипотезу в части сравнения выборок и оказались ей противоположны в части сравнения типов задач. Анализ причин этого результата выходит за рамки данной статьи.

Полученные нами данные позволяют говорить об эвристичности феноменологической модели состоятельности в целом. Об этом свидетельствуют нормальное распределение операциональных показателей модели, их осмысленные и в целом предсказуемые различия в зависимости от выборки и от типов задач и корреляции с более традиционно изучаемыми переменными. Рассмотрение поведения и интерпретация смысла отдельных показателей феноменологической модели представляют собой отдельную задачу. Модель состоятельности требует 
уточнения и «достройки» для интерпретации и прогнозирования активности индивидов, чья жизнь в большей или меньшей степени регламентирует свободу выбора решаемых задач. Возможно, развитие модели потребует использования ранее предложенных ее автором схем «субъектных сборок», построения композиций взаимодействующих субъектов, образующих «внутреннее пространство» личности (Петровский, 2013).

\section{Литература}

Леонтьев, Д. А. (2004). На пути к счастью. В кн. Е. Ананьева (ред.), Энциклопедия для детей (т. 18, с. 545-559). М.: Аванта ${ }^{+}$.

Леонтьев, Д. А. (2006). Личностный потенциал как потенциал саморегуляции. В кн. Б. С. Братусь, Е. Е. Соколова (ред.), Уиеные записки кафедры общей психологии МГУ им. М. В. Ломоносова (вып. 2, с. 85-105). М.: Смысл.

Леонтьев, Д. А. (2011). Саморегуляция как предмет изучения и как объяснительный принщип. В кн. В. И. Моросанова (ред.), Психология саморегуляици в ХХІ в. (с. 74-89). СПб./ М.: Нестор-История. Лефевр, В. А. (1996). Космический субъект. М.: Ин-кварто.

Лефевр, В. А. (2004). Алгебра совести. М.: Когито-центр.

Моросанова, В. И. (2012). Психология саморегуляции. М./СПб.: Нестор-История.

Осин, Е. Н., Леонтьев, Д. А. (2008). Апробация русскоязычных версий двух шкал экспресс-оценки субъективного благополучия. В кн. Материаль ІІІ Всероссийского социологического конгресса. М.: Институт социологии РАН/Российское общество социологов, 2008.

Петровский, В. А. (2010). Человек над ситуащией. М.: Смысл.

Петровский, В. А. (2013). «Я» в персонолоической перспективе. М.: Издательский дом Высшей школы экономики.

Шварцер, Р., Ерусалем, М., Ромек, В. (1996). Русская версия шкалы общей самоэффективности Р. Шварцера и М. Ерусалема. Иностранная психология, 7, 71-76.

Ссылки на зарубежные источники см. в разделе References после англоязынного блока.

Приложение

Методика В.А. Петровского «Психологическая состоятельность личности»

\section{Опросник Вадима Петровского «Задача дня»}

Всю жизнь мы только и делаем, ито решаем задачи. Простье и сложные; бытовые и профессиональные; занимательные и не очень. Ради денег. Ради признания. Или просто потому, ито хочется занять себя чем-то. Некоторые задачи, как говорят, «ставит сама жизнь, а некоторые мы выбираем сами, по своему усмотрению.

Допустим, речь идет о вполне конкретной задаче, которую вы решаете сегодня или, может быть, в последнее время. Попробуйте ответить на ряд вопросов (даже если они покажутся вам не совсем обычными). 
КАК ЧАСТО ПРИ РЕШЕНИИ «ЗАДАЧИ ДНЯ» ВЫ:

1. Перепроверяете себя, пытаетесь удостовериться в правильности своих догадок и предположений (как бы «испытывая их на прочность»)?

(поставьте крестик «х» на шкале «редко - часто»):

редко ----------- часто

2. Считаете нужным дождаться «того самого» счастливого случая, когда ситуация сама подскажет, как быть и что делать (полагаетесь на удачное стечение обстоятельств, как бы наталкивающее на открытие)?

редко часто

3. Исходите из необходимости «отмести» версии, кажущиеся вам сомнительными? редко часто

4. Думаете: вот бы пришел кто-нибудь и сказал, что делать дальше (да только, увы, не будет этого)?

редко ---------------------------------------------- часто

5. Верите в то, что не совершите ошибки, действуя так, а не как-то иначе? редко --------------------------------------- часто

6. Отказываетесь решать задачу по-старому, «отстраиваетесь» от ситуации, внутренне подготавливаетесь к новому решению («переключаетесь»)?

редко ------------------------------------------ часто

7. Вам не хочется ничего делать, нет никаких идей, не испытываете ничего, кроме ощущения полной бесперспективности?

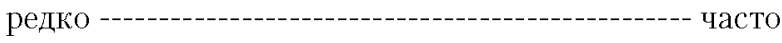

8. Думаете: «Надо попробовать!», хотя и чувствуете, что это совершенно напрасно? редко ------------------- часто

\section{СПАСИБО ЗА ВАШИ ОТВЕТЬ!}

Шкалы второй формы опросника («Задача по вкусу») ничем не отличаются от шкал первой формы («Задача дня»). Отличие в том, что испытуемый, согласно инструкции, имеет в виду задачу, наиболее полно отвечающую его интересами и склонностям. 


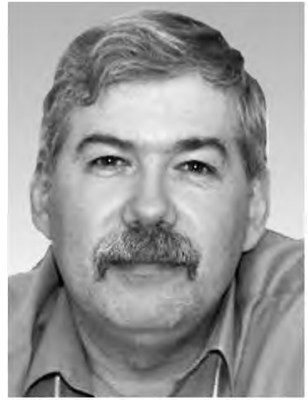

Леонтьев Дмитрий Алексеевич - заведующий лабораторией, Международная лаборатория позитивной психологии личности и мотивации; профессор, департамент психологии, факультет социальных наук, Национальный исследовательский университет «Высшая школа экономики», доктор психологических наук, профeccop.

Сфера научных интересов: психология мотивации, психодиагностика, философская антропология, психология личности.

Контакты: dleontiev@hse.ru

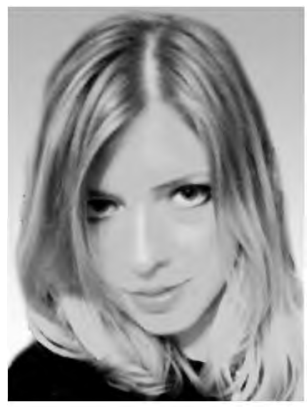

Кифак Лидия Сергеевна - выпускница МГУ имени М.В. Ломоносова, независимый исследователь.

Сфера научных интересов: психология личности, педагогика, психология искусства, психология мотивации, анатомия, изобразительное искусство.

Контакты: manatkina.ls@gmail.com

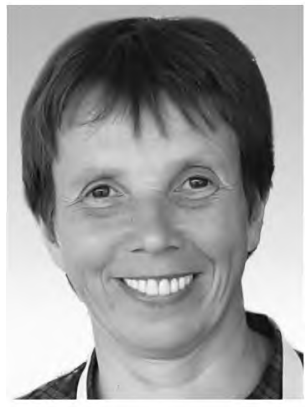

Митина Ольга Валентиновна - ведущий научный сотрудник, факультет психологии, МГУ имени М.В. Ломоносова, кандидат психологических наук.

Сфера научных интересов: математическая психология, психосемантика, политическая психология, саморегуляция.

Контакты: omitina@inbox.ru

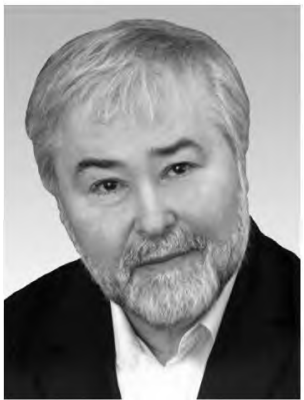

Петровский Вадим Артурович - профессор, кафедра психологии личности, департамент психологии, факультет социальных наук; академический руководитель образовательной программы «Консультативная психология. Персонология», Национальный исследовательский университет «Высшая школа экономики», доктор психологических наук, профессор.

Сфера научных интересов: история, теория и методология психологии, психология личности.

Контакты: petrowskiy@mail.ru 


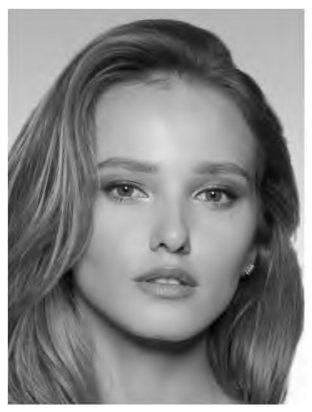

Тихобразова Жанна Сергеевна - выпускница МГУ имени М.В. Ломоносова, независимый исследователь.

Сфера научных интересов: психология личности, психология мотивации, клиническая психология.

Контакты: Zhanna.tia@gmail.com

\title{
Possibleness as a Psychological Construct: Psychometric Validation
}

\author{
D.A. Leontiev ${ }^{\mathrm{a}}$, L.S. Kifak ${ }^{\mathrm{b}}$, O.V. Mitina ${ }^{\mathrm{b}}$, V.A. Petrovsky ${ }^{\mathrm{a}}$, Zh.S. Tikhobrazova ${ }^{\mathrm{b}}$ \\ ${ }^{a}$ National Research University Higher School of Economics, 20 Myasnitskaya Str., Moscore, 101000, \\ Russian Federation \\ ${ }^{b}$ Lomonosov Moscow State University, GSP-1, Leninskie Gory, Moscore, 119991, Russian Federation
}

\begin{abstract}
The objective of the present study is the psychometrical approbation of the inventory "Psychological Possibleness of Personality" (PPP) by V.A. Petrovsky, based on his metaimplicative model of existential choice and Boolean algebra (Petrovsky, 2002). The study was conducted on two samples: freshmen psychology students $(\mathrm{N}=88)$ and professional models $(\mathrm{N}=57)$, who were close on socio-demographics, but radically differed on life style and demands for self-regulation. Besides the Psychological Possibleness of Personality inventory, traditional psychometric inventories for study of personality potential were used: Satisfaction with Life Scale by Diener, General Self-Efficacy Scale by Schwarzer, etc. Six measures of the inventory for two types of tasks were compared, as well as for two samples; analysis of variance for these two variables on the measures of PPP was conducted, as well as correlation analysis of measures of PPP and the two additional scales. It was found that in the sample of models no measure of PPP differs significantly for the two types of tasks, while in the sample of students significant (even highly significant, in most cases) differences are found for all measures, except for the availability of the task. In students correlations of all measures of PPP with satisfaction with life don't differ significantly for the two types of tasks. Their intention and voluntarism are connected with satisfaction with life positively, with judgment and self-control negatively, while connection with availability and attitude is non-significant. In models there are much less significant correlations; an exception is a significant positive correlation with judgment and a negative one with voluntarism for a task "for one's liking". The results allow to speak of the heuristicity of the phenomenological model of possibleness and the construct validity of the inventory PPP.
\end{abstract}

Keywords: self-regulation, psychological possibleness, metaimplicative model, satisfaction with life, self-efficacy. 


\section{References}

Atkinson, J. (1957). Motivational determinants of risktaking behavior. Psychological Review, 64(6), 359-372.

Carver, C., \& Scheier, M. (1998). On the self-regulation of behavior. New York: Cambridge University Press.

Diener, E., Emmons, R. A., Larsen, R. J., \& Griffin, S. (1985). The Satisfaction With Life Scale. Joumal of Personality Assessment, 49, 71-75.

Lefebvre, V. A. (1996). Kosmicheskii sub"ekt [A cosmic subject]. Moscow: In-kvarto.

Lefebvre, V. A. (2004). Algebra sovesti [Algebra of conscience]. Moscow: Kogito-tsentr.

Leontiev, D. A. (2004). Na puti $k$ schast'yu [One the way to happiness]. In E. Ananieva (Ed.), Entsiklopediya dlya detei [Encyclopedia for children] (Vol. 18, pp. 545-559). Moscow: Avanta ${ }^{+}$.

Leontiev, D. A. (2006). Lichnostnyi potentsial kak potentsial samoregulyatsii [Personality potential as a potential of self-regulation]. In B. S. Bratus' \& E. E. Sokolova (Eds.), Uchemye zapiski kafedry obshchei psikhologii MGU im. M. V. Lomonosova [Proceedings of the chair of general psychology of Lomonosov MSU] (Iss. 2, pp. 85-105). Moscow: Smysl.

Leontiev, D. A. (2011). Samoregulyatsiya kak predmet izucheniya i kak ob"yasnitel'nyi printsip [Selfregulation as a subject matter and as an explanatory principle]. In V. I. Morosanova (Ed.), Psikhologiya samoregulyatsii v XXI v. [Psychology of self-regulation in the XXI century] (pp. 74-89). Saint Petersburg/Moscow: Nestor-Istoriya.

Morosanova, V. I. (2012). Psikhologiya samoregulyatsii [Psychology of self-regulation]. Moscow/Saint Petersburg: Nestor-Istoriya.

Osin, E. N., \& Leontiev, D. A. (2008). Aprobatsiya russkoyazychnykh versii dvukh shkal ekspressotsenki sub"ektivnogo blagopoluchiya [Approbation of the Russian versions of two scales for express-assessment of subjective well-being]. In Materialy III Vserossiiskogo sotsiologicheskogo kongressa [Proceedings of the III All-Russian sociological congress]. Moscow: Institut sotsiologii RAN/Rossiiskoe obshchestvo sotsiologov, 2008.

Petrovsky, V. A. (2010). Chelovek nad situatsiei [A man above situation]. Moscow: Smysl.

Petrovsky, V. A. (2013). "Ya" v personologicheskoi perspective ["I" in personological perspective] Moscow: HSE Publishing House.

Schwartzer, R. (1993). Measurement of perceived self-efficacy. Psychometric scales for cross cultural research. Berlin: Freie Universitat Berlin/Institut für Psychologie.

Schwarzer, R., Jerusalem, M., \& Romek, V. (1996). Russkaya versiya shkaly obshchei samoeffektivnosti R. Shvartsera i M. Erusalema [The Russian version of the General Self-Efficacy scaly by R. Schwarzer and M. Jerusalem]. Inostrannaya Psikhologiya, 7, 71-77.

Dmitry A. Leontiev - head of International Laboratory of Positive Psychology of Personality and Motivation; professor, School of Psychology, Faculty of Social Sciences, National Research University Higher School of Economics, D.Sc.

Research area: psychology of motivation, psychodiagnostics, philosophical anthropology, psychology of personality.

E-mail: dleontiev@hse.ru 
Lidia S. Kifak - independent researcher.

Research area: psychology of personality and motivation, pedagogy, psychology of art, anatomy, fine arts.

E-mail: manatkina.ls@gmail.com

Olga V. Mitina - leading research fellow, Deptartment of Psychology, Lomonosov Moscow State University, Ph.D.

Research area: mathematical psychology, psychosemantics, political psychology, self-regulation. E-mail: omitina@inbox.ru

Vadim A. Petrovsky - professor, Department of Psychology of Personality, School of Psychology, Faculty of Social Sciences; programme academic supervisor «Counselling Psychology. Personality Studies», National Research University Higher School of Economics, D.Sc

Research area: history, theory and methodology of psychology, psychology of personality. E-mail: petrowskiy@mail.ru

Zhanna S. Tikhobrazova - independent researcher.

Research area: psychology of personality and motivation, clinical psychology.

E-mail: Zhanna.tia@gmail.com 